Notfall Rettungsmed 2017 $\cdot 20: 188-189$

DOI 10.1007/s10049-017-0300-z

Online publiziert: 19. April 2017

(c) Springer Medizin Verlag GmbH 2017

CrossMark

\section{Dodt ${ }^{1} \cdot$ C. Wrede ${ }^{2}$}

'Notfallzentrum am Klinikum Bogenhausen, Klinikum München, München, Deutschland

${ }^{2}$ Interdisziplinäre Notfallzentrum, HELIOS Klinikum Berlin-Buch, Berlin, Deutschland

\title{
Infektiologie und Hygiene bei Notfallpatienten
}

Liebe Leserinnen und Leser,

im Eckpunktepapier 2016 zur notfallmedizinischen Versorgung [1] wurde erstmals die Sepsis als eine neue Tracerdiagnose aufgenommen. Damit steht neben den klar umschriebenen Notfalldiagnosen wie Herzinfarkt, Polytrauma und Schlaganfall erstmals ein Syndrom mit oft schleichendem Beginn und unscharfer Symptomatik sowie versteckter Ursache in der Liste der Krankheitszustände, für die in der notfallmedizinischen Versorgung besondere Aufmerksamkeit gefordert wird. Die Sepsis als infektionsgetriggertes Inflammationssyndrom ist Endstrecke jeder schweren Infektion und hat eine sehr hohe Krankenhausmortalität. Damit wird klar, welche enorme Herausforderung jede Infektion für die Notfallmediziner darstellen kann, insbesondere, wenn sie in der Notaufnahme die ersten diagnostischen und therapeutischen Schritte einleiten. Diese ersten Schritte sind oft entscheidend für den weiteren therapeutischen Erfolg. Das Erkennen der Infektion und die Erfassung des Schwergrads, die Einleitung korrekter diagnostischer Schritte und der unmittelbare Beginn einer adäquaten Therapie unter Berücksichtigung des Infektionsfokus und der Wirtsfaktoren sind die wesentlichen Garanten einer erfolgreichen Therapie.

Jeder Notfallmediziner muss die Grundzüge der Infektiologie beherrschen, die ihn in die Lage versetzen, bereits in der Notaufnahme unter Zeitdruck die richtigen Weichen für die weiteren Therapien zu stellen. Die aktuelle Ausgabe von Notfall+Rettungsmedizin hat einige Aspekte aus der Infektiologie für Notfallmediziner zusammengestellt. Einige Artikel wie der Beitrag zur An- tibiotikatherapie von Hitzenbichler et al. sind von zentraler Bedeutung für jeden an einer bakteriellen Infektion erkrankten Patienten. Andere Beiträge wiederum betrachten besondere Patientengruppen, wie Patienten mit Transplantationen $(\mathrm{Ca}$ kiroglu et al.), onkologische Patienten (Schmidt-Hieber et al.) und Flüchtlinge (Seilmaier et al.), die zwar nur einen kleinen Anteil der Notfallpatienten ausmachen, aber durch eine ausgeprägte Immunsuppression oder bei uns wenig bekannte Erreger besonders gefährdet sind und besonderer Kenntnisse bedürfen. Schließlich wird der wichtige Aspekt der Infektionsprophylaxe bei der großen Gruppe der Patienten mit offenen Wunden von Leidel et al. behandelt.

\section{\) Notfallmediziner müssen vor Einleitung der Therapie die Grundzüge der Infektiologie beherrschen}

Wie steht es nun mit der Qualität der Infektionserkennung und -therapie in deutschen Notaufnahmen? Das ist eine Frage, die man in Deutschland nicht gut beantworten kann, denn aussagekräftige Statistiken diesbezüglich werden nicht geführt. Die Initiativen zur Qualitätssicherung in der stationären Versorgung betrachtet zwar die Versorgungswirklichkeit von Patienten z.B. mit einer ambulant erworbenen Pneumonie, aber hier werden nur Patienten mit einer stationären Aufnahmeindikation betrachtet, ambulant behandelte Patienten werden nicht eingeschlossen. Es muss in diesem Zusammenhang auch kritisch hinterfragt werden, ob es wirklich ein Zeichen guter Qualität ist, wenn die
Erstantibiose innerhalb von $8 \mathrm{~h}$ verabreicht werden darf, so wie es in der Qualitätsinitiative abgefragt wird. In gut organisierten Notaufnahmen sollte die Erstantibiose spätestens innerhalb von $2 \mathrm{~h}$ appliziert worden sein, bei septischen Patienten ist eine noch kürzere „doorto-antibiotic time" anzustreben. Bei der Betrachtung der Statistiken der Qualitätssicherungsinitiativen fällt eine sehr große Streuung bezüglich der Qualitätsindikatoren für eine gute Prozessqualität in der Aufnahmesituation wie die Bestimmung der Atemfrequenz, der Sauerstoffsättigung und der zeitgerechten Antibiotikagabe auf, wobei Zentren mit hohen Fallzahlen überdurchschnittlich gute Ergebnisse aufweisen. Das spricht für eine Zentralisierung auch im Bereich der Notfallmedizin.

Die Tatsache, dass die Notaufnahmen eine hohe Zahl akuter Infektionen zu behandeln haben und in der Diagnostik und Therapie entscheidende Weichen stellen, legt die Einbindung von Notaufnahmen in die Infektionssurveillance nahe. Eine Häufung schwerer Infektionen wird sich sofort in einem deutlichen Anstieg der Fallzahl der Notaufnahmen widerspiegeln, wie durch das AKTIN-Projekt bzw. das Nationale Notaufnahmeregister exemplarisch gezeigt werden konnte [2]. In der Arbeitsgemeinschaft Influenza des Robert Koch-Instituts (RKI) sind Notaufnahmen als Sentinelzentren bisher nicht angefragt worden.

Von guten infektiologischen Kenntnissen in der klinischen Notfallmedizin profitieren sicherlich die Patienten ebenso wie alle Abteilungen eines Krankenhauses. Eine rasche, zielführende Diagnostik und eine rationelle empirische, korrekt dosierte antibiotische Therapie nach dem bereits von Paul Ehrlich aufge- 
stellten Prinzip „frapper fort et frapper vite" auf dem Boden der lokalen Resistenzstatistiken wird den weiteren Behandlungsverlauf positiv beeinflussen und die Liegedauer und Mortalität reduzieren.

Wenn die aktuelle Ausgabe von Notfall+Rettungsmedizin dazu beitragen kann, die Versorgung von Patienten mit Infektionserkrankungen zu verbessern, würden wir uns sehr freuen! Wir wünschen Ihnen viel Vergnügen und neue Erkenntnisse bei der Lektüre dieser Ausgabe.

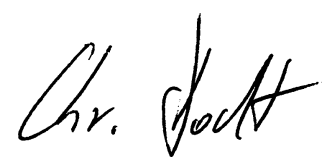

Prof. Dr. C. Dodt

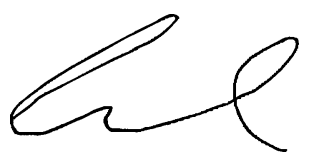

Prof. Dr. C. Wrede

\section{Korrespondenzadresse}

\section{Prof. Dr. med. C. Dodt}

Notfallzentrum am Klinikum Bogenhausen, Klinikum München

Englschalkinger Straße 77, 81925 München, Deutschland

christoph.dodt@klinikum-muenchen.de

\section{Prof. Dr. med. C. Wrede}

Interdisziplinäre Notfallzentrum, HELIOS

Klinikum Berlin-Buch

Schwanebecker Chaussee 50, 13125, Berlin,

Deutschland

christian.wrede@helios-kliniken.de

Interessenkonflikt. C. Dodt und C. Wrede geben an, dass kein Interessenkonflikt besteht.

\section{Literatur}

1. Fischer $\mathrm{M}$, Kehrberger E, Marung $\mathrm{H}$ et al (2016) Eckpunktepapier 2016 zur notfallmedizinischen Versorgung der Bevölkerung in der Prähospitalphase und in der Klinik. Notfall Rettungsmed 19(5):387-395. doi:10.1007/s10049-016-0187-0 2. Greiner F, Ziehm D, Rexroth U et al. Surveillance of acute respiratory infections (ARI) through usage of emergency department data. https:// aktin.org/images/pdf/HEC-2016_v4_eng-A0.pdf. Zugegriffen: 10.04.2017

Hier steht eine Anzeige.

\section{Springer}

